\title{
Flatness-based Control and Conventional RST Polynomial Control of a Thermal Process
}

\author{
Hajer Gharsallaoui, Mounir Ayadi, Mohamed Benrejeb, Pierre Borne
}

Hajer Gharsallaoui

École Nationale d'Ingénieurs de Tunis, LAboratoire de Recherche en Automatique (LA.R.A.)

B.P. 37, le Belvédère, 1002 Tunis, Tunisia

Laboratoire d'Automatique, Génie Informatique et Signal (LAGIS), École Centrale de Lille

Avenue Paul Langevin BP 4859651 VILLENEUVE D’ASCQ CEDEX, France

E-mail: hajer.gharsallaoui@voila.fr

\begin{abstract}
In this paper, a comparison between conventional RST polynomial control by poles placement and RST flatness-based control is proposed. These approaches were developed, in discrete-time formalism by carrying out a generation of a reference starting from a flat output and by then analyzing the tracking error in closedloop. The case of the thermal system, that we applied an output disturbance, was considered by simulation to study the effectiveness of given flatness-based robust controller with the integration of an anti-windup device, in terms of tracking trajectory and disturbance rejection.

Keywords: Flatness, RST control, tracking trajectory, robustness, anti-windup.
\end{abstract}

\section{Introduction}

The flatness property was introduced by M. Fliess, J. Lévine, P. Martin and P. Rouchon in 1992 to propose a new strategy to control continuous nonlinear systems with good performances in term of tracking trajectory. At first, the use of this property consists in the definition of an output trajectory allowing the determination of the variables of the flat system. Secondly, it concerns the elaboration of the control in closed- loop allowing to obtain a stable system giving place to a tracking of a desired trajectory with an error which tends asymptotically towards zero $[1,2,5,18,19]$.

Several techniques of control resulting from the theory of control were established and applied to real systems, especially the RST polynomial control. The exploitation of robust control with an argued choice for its design satisfying the control objectives in term of the tracking trajectory of reference as well as the regulation in the presence of disturbances, constitute the object of this paper.

A comparison between this well-known conventional RST polynomial control and a recent approach of a RST controller design based on the property of flatness of the mono-variable linear and controllable systems are carried out by considering the case of the thermal process control.

\section{Conventional RST polynomial control}

\subsection{Structure of RST controller by poles placement}

In the RST control approach it is possible to impose poles in closed-loop and to carry out in a separate way the objectives of tracking and regulation. In a discrete formalism, the blocks of RST controller are given by figure $1,[4,9,12]$. 


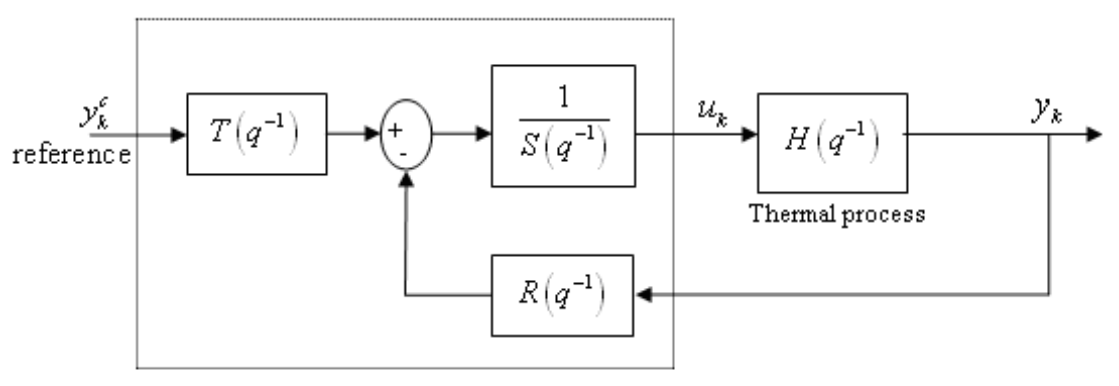

RST Controller

Figure 1: Conventional structure RST controller

$H\left(q^{-1}\right)=\frac{B\left(q^{-1}\right)}{A\left(q^{-1}\right)}$ is the transfer function of the dynamic linear discrete system where the polynomials $A$ and $B$ represent respectively the denominator and the numerator of the transfer function with respective degrees $n_{A}$ and $n_{B}$ :

$$
\begin{aligned}
& A\left(q^{-1}\right)=a_{0}+a_{1} q^{-1}+\ldots+a_{n_{A}} q^{-n_{A}} \\
& B\left(q^{-1}\right)=b_{0}+b_{1} q^{-1}+\ldots+b_{n_{B}} q^{-n_{B}}
\end{aligned}
$$

The choice of the polynomials $R, S$ and $T$ allows to solve as well the problem of regulation as well as of tracking. These polynomials are given by:

$$
\begin{aligned}
& R\left(q^{-1}\right)=r_{0}+r_{1} q^{-1}+\ldots+r_{n_{R}} q^{-n_{R}} \\
& S\left(q^{-1}\right)=s_{0}+s_{1} q^{-1}+\ldots+s_{n_{S}} q^{-n_{S}} \\
& T\left(q^{-1}\right)=t_{0}+t_{1} q^{-1}+\ldots+t_{n_{T}} q^{-n_{T}}
\end{aligned}
$$

where $n_{R}=\operatorname{deg}(R), n_{S}=\operatorname{deg}(S)$ and $n_{T}=\operatorname{deg}(T)$. The transfer function in closed-loop, in this case, is given by:

$$
H_{B F}\left(q^{-1}\right)=\frac{T\left(q^{-1}\right) B\left(q^{-1}\right)}{A\left(q^{-1}\right) S\left(q^{-1}\right)+B\left(q^{-1}\right) R\left(q^{-1}\right)}
$$

The calculation of the polynomials $R$ and $S$ is carried out starting from the choice of the polynomial $P$ :

$$
\begin{gathered}
P\left(q^{-1}\right)=A\left(q^{-1}\right) S\left(q^{-1}\right)+B\left(q^{-1}\right) R\left(q^{-1}\right) \\
=p_{0}+p_{1} q^{-1}+\cdots+p_{n_{p}} q^{-n_{p}}
\end{gathered}
$$

If $\operatorname{deg}(P)<\operatorname{deg}(A)+\operatorname{deg}(B)$, this equation is regular and it can be written in the following matrix form:

$$
\underbrace{\left(\begin{array}{cccccccc}
a_{0} & 0 & \cdots & 0 & b_{0} & 0 & \cdots & 0 \\
a_{1} & a_{0} & \ddots & \vdots & b_{1} & b_{0} & \ddots & \vdots \\
\vdots & \ddots & \ddots & 0 & \vdots & \ddots & \ddots & 0 \\
a_{n_{A}} & \vdots & \ddots & a_{0} & b_{n_{B}} & \vdots & \ddots & b_{0} \\
0 & \ddots & \vdots & a_{1} & 0 & \ddots & \vdots & b_{1} \\
\vdots & \ddots & \ddots & \vdots & \vdots & \ddots & \ddots & \vdots \\
0 & \cdots & \cdots & a_{n_{A}} & 0 & \cdots & \cdots & b_{n_{B}}
\end{array}\right)}_{M} \underbrace{\left(\begin{array}{c}
s_{0} \\
\vdots \\
s_{n_{S}} \\
r_{0} \\
\vdots \\
\vdots \\
r_{n_{R}}
\end{array}\right)}_{x}=\underbrace{\left(\begin{array}{c}
p_{0} \\
p_{1} \\
\vdots \\
p_{n_{P}} \\
0 \\
\vdots \\
0
\end{array}\right)}_{M_{P}}
$$


where $M \in \mathfrak{R}^{\left(n_{A} \times n_{B}\right)} \times \mathfrak{R}^{\left(n_{A} \times n_{B}\right)}$ is called Sylvester matrix. The coefficients of $R\left(q^{-1}\right)$ and $S\left(q^{-1}\right)$ contained in vector $x$ are given by:

$$
x=M^{-1} M_{P}
$$

For the determination of the coefficients of the polynomial $T$, in the case of the tracking of a desired trajectory $y_{k}^{c}$ corresponding to a reference model:

$$
H_{m}\left(q^{-1}\right)=\frac{B_{m}\left(q^{-1}\right)}{A_{m}\left(q^{-1}\right)}
$$

we choose $T\left(q^{-1}\right)=\beta B\left(q^{-1}\right)$, to obtain:

$$
\begin{gathered}
H_{B F 1}\left(q^{-1}\right)=\beta B\left(q^{-1}\right) \\
H_{B F}\left(q^{-1}\right)=\beta \frac{B\left(q^{-1}\right) B_{m}\left(q^{-1}\right)}{A_{m}\left(q^{-1}\right)}
\end{gathered}
$$

with taking $\beta=\frac{1}{B(1)}$, a static gain in closed-loop equal to 1 is then imposed.

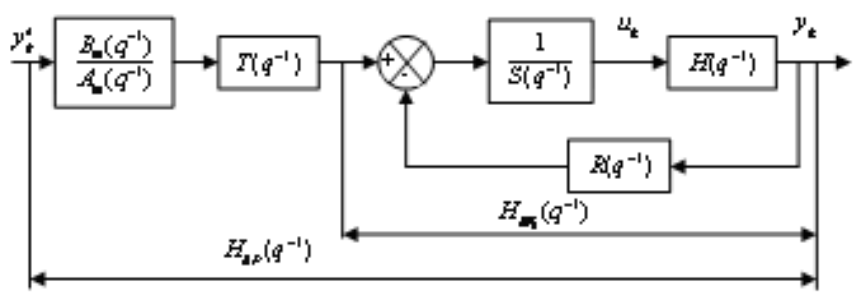

Figure 2: Detailed structure of RST regulator

\subsection{Rejection of disturbances}

Let us consider $S\left(q^{-1}\right)$ the polynomial of the following form:

$$
S\left(q^{-1}\right)=\left(1-q^{-1}\right)^{m} S^{\prime}\left(q^{-1}\right)
$$

To reject a step disturbance, we can choose $m=1$ with $\operatorname{deg}\left(S^{\prime}\right)=\operatorname{deg}(S)-m$.

\subsection{Anti-saturation of the control}

The direct implementation of RST controller often leads to a control signal whose amplitude can be very important. In order to keep this amplitude in an acceptable interval $\left[u_{\min }, u_{\max }\right]$, we add to the structure of control an anti-windup device [4], $d$ is the output disturbance and $S^{*}\left(q^{-1}\right)$ is a polynomial defined from the relation bellow:

$$
S\left(q^{-1}\right)=s_{0}+q^{-1} S^{*}\left(q^{-1}\right)
$$




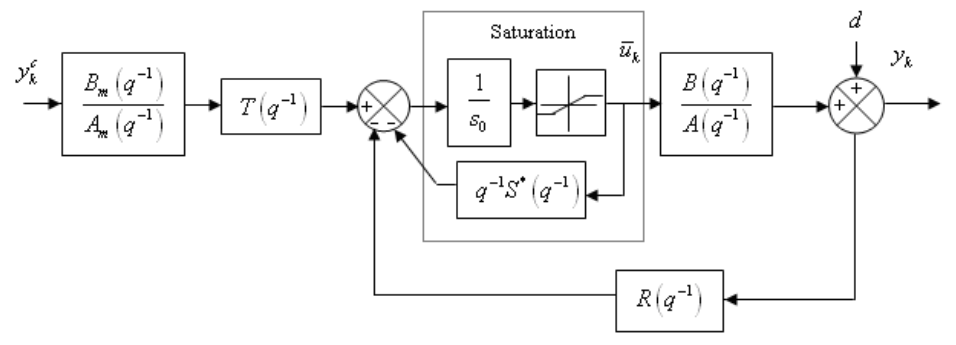

Figure 3: Conventional structure of RST controller with anti-windup device

\section{Design of flatness-based controller}

\subsection{Determination of flat output}

In this part, let us consider the discrete-time linear system Single Input Single Output (SISO), described by the following equation:

$$
A\left(q^{-1}\right) y_{k}=B\left(q^{-1}\right) u_{k}
$$

where $y_{k}$ and $u_{k}$ represent the output and the control signals respectively.

The flat output is a variable defined in continuous time; we consider the expressions of the polynomials $A\left(q^{-1}\right)$ and $B\left(q^{-1}\right)$ in term of the operator $q^{-1}$, to be able to define thereafter the discrete flat output of the system.

The system is considered as linear and controllable, consequently it is flat and its flat output $z_{k}$ is given by [2]:

$$
z_{k}=N\left(q^{-1}\right) y_{k}+D\left(q^{-1}\right) u_{k}
$$

where $N\left(q^{-1}\right)$ and $D\left(q^{-1}\right)$ are polynomials which represent the solutions of the equation of Bezout:

$$
A\left(q^{-1}\right) D\left(q^{-1}\right)+B\left(q^{-1}\right) N\left(q^{-1}\right)=1
$$

The flat output $z_{k}$ on which depend the output $y_{k}$ and the control $u_{k}$, can be also seen as being the partial state of a linear system [3]:

$$
\begin{aligned}
& u_{k}=A\left(q^{-1}\right) z_{k} \\
& y_{k}=B\left(q^{-1}\right) z_{k}
\end{aligned}
$$

The knowledge of the flat output $z_{k}$ allows to determine the open loop control $u_{k}$ of the system.

\subsection{Trajectories planning}

The objective of the trajectories planning [17] is to determine an open-loop control $u^{d}(t)$, carrying out the objective bringing a given system, of a certain initial state in a known final state:

$$
\begin{gathered}
u^{d}(t)=\mathrm{B}\left(z^{d}(t), \ldots, z^{d(\alpha+1)}(t)\right) \\
y^{d}(t)=\mathrm{C}\left(z^{d}(t), \ldots, z^{d(\sigma)}(t)\right)
\end{gathered}
$$


$z^{d}$ is the desired trajectory for the flat output that is $\sup (\alpha+1, \sigma)$ time continuously derivable.

If the objective is to reach two equilibrium points of balance:

$\left(u\left(t_{0}\right), y\left(t_{0}\right), z\left(t_{0}\right)\right)$ and $\left(u\left(t_{f}\right), y\left(t_{f}\right), z\left(t_{f}\right)\right)$, where $t_{0}$ and $t_{f}$ are the two times known in advance, it is necessary to solve the problem of trajectory generation offline. A solution for the calculation of $Z^{d}(t)$, vector of $z^{d}(t)$ and its successive derivatives, is given by the relation:

$$
Z^{d}(t)=M_{1}\left(t-t_{0}\right) c_{1}+M_{2}\left(t-t_{0}\right) c_{2}
$$

where $M_{1}(t)$ and $M_{2}(t)$ are the two following matrices:

$$
\begin{gathered}
M_{1}(t)=\left(\begin{array}{cccc}
1 & t & \cdots & \frac{t^{n-1}}{(n-1) !} \\
0 & 1 & \cdots & \frac{t^{n-2}}{(n-2) !} \\
\vdots & \ddots & \ddots & \vdots \\
0 & \cdots & 0 & 1
\end{array}\right) \\
M_{2}(t)=\left(\begin{array}{cccc}
\frac{t^{n}}{n !} & \frac{t^{n+1}}{(n+1) !} & \cdots & \frac{t^{2 n-1}}{(2 n-1) !} \\
\frac{t^{n-1}}{(n-1) !} & \frac{t^{n}}{n !} & \cdots & \frac{t^{2 n-2}}{(2 n-2) !} \\
\vdots & \ddots & \ddots & \vdots \\
t & \cdots & \cdots & \frac{t^{n}}{n !}
\end{array}\right)
\end{gathered}
$$

The vectors $c_{1}$ and $c_{2}$ can be defined from 20 and 21 by:

$$
\begin{gathered}
c_{1}=Z^{d}\left(t_{0}\right) \\
c_{2}=M_{2}^{-1}\left(t_{f}-t_{0}\right)\left(Z^{d}\left(t_{f}\right)-M_{1}\left(t_{f}-t_{0}\right) Z^{d}\left(t_{0}\right)\right)
\end{gathered}
$$

In the discrete-time formalism, the real output of system $y_{k}$ to be controlled, follows the desired trajectory $y_{k}^{d}$ expressed by:

$$
y_{k}^{d}=B\left(q^{-1}\right) z_{k}^{d}
$$

The synoptic diagram of figure 5 summarizes the different steps as well as the necessary tools to generate the desired trajectory $y_{k}^{d}$ in a discrete-time formalism with considering the sampling period $T_{e}$ $[2,5,12]$.

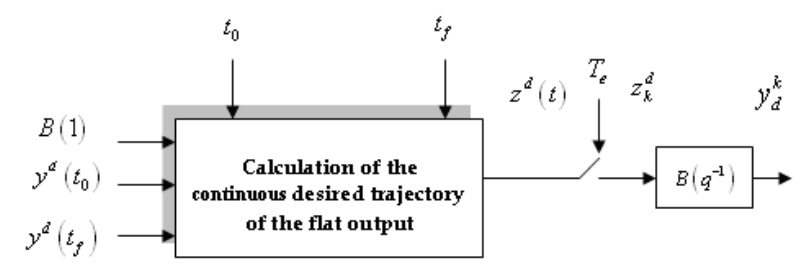

Figure 4: Generation of the desired trajectory $y_{k}^{d}$

\subsection{Flatness-based RST polynomial controller}

The realization of flatness RST controller is carried out here by considering the method of direct calculation of the state vector $Z_{k}=\left(\begin{array}{llll}z_{k} & z_{k+1} & \cdots & z_{k+n-1}\end{array}\right)^{T}$, suggested in [2, 5]. 
From the relations (20) and (21), we can determine the representation of state of the system in its controllable form:

$$
\left\{\begin{array}{l}
Z_{k+1}=\mathbf{A} Z_{k}+\mathbf{B} u_{k} \\
y_{k}=\mathbf{C} Z_{k}
\end{array}\right.
$$

with:

$$
\begin{aligned}
& \mathbf{A}=\left(\begin{array}{ccccc}
0 & 1 & 0 & \cdots & 0 \\
0 & \ddots & 1 & \ddots & \vdots \\
\vdots & \ddots & \ddots & \ddots & 0 \\
0 & \cdots & 0 & 0 & 1 \\
-a_{0} & -a_{1} & \cdots & -a_{n-2} & -a_{n-1}
\end{array}\right), \mathbf{B}=\left(\begin{array}{c}
0 \\
\vdots \\
0 \\
1
\end{array}\right) \text { and } \\
& \mathbf{C}=\left(\begin{array}{cccc}
b_{0} & b_{1} & \cdots & b_{n-1}
\end{array}\right)
\end{aligned}
$$

From this representation, we can write [5]:

$$
Z_{k}=O_{(\mathbf{A}, \mathbf{C})}^{-1}\left(Y_{k}-M_{(\mathbf{A}, \mathbf{B}, \mathbf{C})} U_{k}\right)
$$

with the observability matrix is:

$$
O_{(\mathbf{A}, \mathbf{C})}=\left(\begin{array}{c}
\mathbf{C} \\
\mathbf{C A} \\
\vdots \\
\mathbf{C A}^{n-1}
\end{array}\right)
$$

and the controllability matrix $M_{(\mathbf{A}, \mathbf{B}, \mathbf{C})}$ given by:

$$
M_{(\mathbf{A}, \mathbf{B}, \mathbf{C})}=\left(\begin{array}{cccc}
0 & \cdots & \cdots & 0 \\
\mathbf{C B} & \ddots & & \vdots \\
\mathbf{C A B} & \mathbf{C B} & \ddots & \vdots \\
\vdots & \ddots & \ddots & 0 \\
\mathbf{C A}^{n-2} \mathbf{B} & \cdots & \mathbf{C A B} & \mathbf{C B}
\end{array}\right)
$$

From [5], we obtain the flatness control law by the following relation [12]:

$$
u_{k}=K(q) z_{k}^{d}+(a-k) Z_{k}
$$

where $a$ and $k$ are two constant vectors constituted by the $a_{i}$ and $k_{i}$ coefficients of the $A\left(q^{-1}\right)$ and $K(q)$ polynomials given by: $a=\left(\begin{array}{llll}a_{0} & a_{1} & \ldots & a_{n-1}\end{array}\right), k=\left(\begin{array}{llll}k_{0} & k_{1} & \ldots & k_{n-1}\end{array}\right)$, where $K(q)$ is a polynomial containing the closed-loop poles. The realisable structure of RST controller by flatness with $q^{-1}$ operator can be then obtained by:

$$
S\left(q^{-1}\right) u_{k}=K(q) z_{k}^{d}+R\left(q^{-1}\right) y_{k}
$$

where:

$$
\begin{gathered}
R\left(q^{-1}\right)=-(a-k) \mathbf{A}^{n-1} O_{(\mathbf{A}, \mathbf{C})}^{-1} Q \\
S\left(q^{-1}\right)=1+(a-k)\left(\mathbf{A}^{n-1} O_{(\mathbf{A}, \mathbf{C})}^{-1} M_{(\mathbf{A}, \mathbf{B}, \mathbf{C})}-\left(\begin{array}{lll}
\mathbf{A}^{n-2} \mathbf{B} & \ldots & \mathbf{B}
\end{array}\right)\right) Q^{*}
\end{gathered}
$$

and:

$Q=\left(\begin{array}{lllll}q^{-(n-1)} & q^{-(n-2)} & \ldots & q^{-1} & 1\end{array}\right)^{T}, Q^{*}=\left(\begin{array}{llll}q^{-(n-1)} & q^{-(n-2)} & \ldots & q^{-1}\end{array}\right)^{T}$.

The dynamics of the closed-loop is defined by the tracking polynomial $K\left(q^{-1}\right)$ such as: 


$$
A\left(q^{-1}\right) S\left(q^{-1}\right)+B\left(q^{-1}\right) R\left(q^{-1}\right)=K\left(q^{-1}\right)
$$

This relation represents the Bezout equation already defined in the traditional approach of RST controller. The choice of poles must be well optimized in order to satisfy the desired performances. This step appears the fundamental contribution of the exploitation of the flatness property in the design of such controller.

Indeed, the choice of the closed-loop poles corresponds to this of the tracking model of a desired trajectory and more precisely, to the poles of the $K(q)$ polynomial.

\section{Study of the robustness}

\subsection{Disturbances rejection and noises attenuation}

By using the principle of the internal model, a deterministic disturbance can be rejected by considering in the transfer function of the direct chain an integrator. In fact, in order to obtain a robust controller, the polynomial $S\left(q^{-1}\right)$ must have a term in $\left(1-q^{-1}\right)$ which allows the rejection of static disturbance present on the output signal. Moreover, to introduce a filtering effect into a certain zone of frequencies, the principle of the blocking of the signal is often used by forcing the system to behave as an open loop system at these frequencies; what is equivalent to impose of the zeros in polynomial $R\left(q^{-1}\right)$. This led so that polynomial $R\left(q^{-1}\right)$ contains a term of $\left(1+q^{-1}\right)$ making it possible to ensure an attenuation of the noises effect in high frequencies on the system input $[4,6]$.

Thus, the following polynomials $H_{S}\left(q^{-1}\right)$ and $H_{R}\left(q^{-1}\right)$ are introduced into the model of the system:

$$
\begin{aligned}
& H_{S}\left(q^{-1}\right)=1-q^{-1} \\
& H_{R}\left(q^{-1}\right)=1+q^{-1}
\end{aligned}
$$

By taking in account of these pre-specified fixed parts, polynomials $\tilde{R}\left(q^{-1}\right)$ and $\tilde{S}\left(q^{-1}\right)$ of new RST controller can be expressed by:

$$
\begin{aligned}
& \tilde{R}\left(q^{-1}\right)=H_{R}\left(q^{-1}\right) R\left(q^{-1}\right) \\
& \tilde{S}\left(q^{-1}\right)=H_{S}\left(q^{-1}\right) S\left(q^{-1}\right)
\end{aligned}
$$

In addition, the dynamics of the regulation is defined by the choice of the dominant and auxiliary poles of polynomial $K\left(q^{-1}\right)$ of the closed loop given by the relation:

$$
A\left(q^{-1}\right) H_{S}\left(q^{-1}\right) S\left(q^{-1}\right)+B\left(q^{-1}\right) H_{R}\left(q^{-1}\right) R\left(q^{-1}\right)=K\left(q^{-1}\right)
$$

In order to take in account the pre-specified parts in the design of the new controller, it is enough to consider the increased model governed by the following transfer function:

$$
\tilde{H}\left(q^{-1}\right)=\frac{B\left(q^{-1}\right) H_{R}\left(q^{-1}\right)}{A\left(q^{-1}\right) H_{S}\left(q^{-1}\right)}
$$

Then, it is a question to redo the calculations of flatness-based RST controller using the method presented previously. 


\subsection{Analyze of the controller robustness}

In order to maintain nominal performances in rejection of disturbances and in the presence of modelling errors, the sensitivity functions defined below, are calibrated and recomputed so as to satisfy the performances required. The sensitivity functions are given according to the type of disturbance to consider. By considering the three types of following disturbances: output-input disturbances and noises of measurement, we can thus deduce the output-input functions of sensitivity described in $[4,6]$ :

- Output disturbance: represented by the transfer function $S_{y d}\left(q^{-1}\right)$ between the output disturbance $d(t)$ and the system output $y(t)$ :

$$
S_{y d}\left(q^{-1}\right)=\frac{A\left(q^{-1}\right) \tilde{S}\left(q^{-1}\right)}{A\left(q^{-1}\right) \tilde{R}\left(q^{-1}\right)+B\left(q^{-1}\right) \tilde{S}\left(q^{-1}\right)}=\frac{A\left(q^{-1}\right) \tilde{S}\left(q^{-1}\right)}{K\left(q^{-1}\right)}
$$

- Input disturbance: represented by the transfer function $S_{u d}\left(q^{-1}\right)$ between the output disturbance $d(t)$ and the input $u(t)$ :

$$
S_{u d}\left(q^{-1}\right)=\frac{-A\left(q^{-1}\right) \tilde{R}\left(q^{-1}\right)}{K\left(q^{-1}\right)}
$$

The study of the robustness of the developed RST controller is based on the frequencies analysis of the modules of the various functions of sensitivity. Desirable templates for the sensitivity functions will be defined through the constraints of imposed performances and tolerated robustness margins. The purpose is to have the disturbance output sensitivity function inside the upper and lower templates as shown further in figures 14 and 15. Also, input sensitivity function must have a decreasing shape meaning that the controller is insensitive to noise.

\section{Saturation effects}

The additive static disturbances cause generally the increase in the amplitudes of the control signal applied to the system exceeding certain limit values. This especially presents a problem in the control of process by digital computers having thresholds in tension not to exceed on the level of their inputoutputs. Consequently, it is necessary to design a device of anti-saturation according to the technique already developed in [4], where the law of control has the following forms:

$$
u_{k}=K(q) z_{k}^{d}-\tilde{R}\left(q^{-1}\right) y_{k}-\bar{S}\left(q^{-1}\right) u_{k-1}
$$

with:

$$
\tilde{S}\left(q^{-1}\right)=1+q^{-1} \bar{S}\left(q^{-1}\right)
$$

In addition, by considering $u_{\min }$ and $u_{\max }$ respectively the lower and higher limits of the control saturation, we obtain:

$$
\bar{u}_{k}=\left\{\begin{array}{l}
u_{k} \text { if } u_{\min } \leq u_{k} \leq u_{\max } \\
u_{\max } \text { if } u_{k}>u_{\max } \\
u_{\min } \text { if } u_{k}<u_{\min }
\end{array}\right.
$$

However, it is possible to impose certain dynamics when the system leaves the saturation. This is illustrated in figure 5. The desired dynamics is defined by the polynomial $P_{S}\left(q^{-1}\right)$ given by equation (48).

$$
P_{S}\left(q^{-1}\right)=1-\exp \left(\frac{T_{e}}{\tau_{\text {sat }}}\right) q^{-1}
$$


where $\tau_{\text {sat }}$ indicates a time-constant of a system of first order. It is it should be noted that outside of saturation, $u_{\min }<u_{k}<u_{\max }$, this mechanism of anti-saturation is equivalent to block $\frac{1}{\tilde{S}\left(q^{-1}\right)}$ of RST controller given by figure 5 .

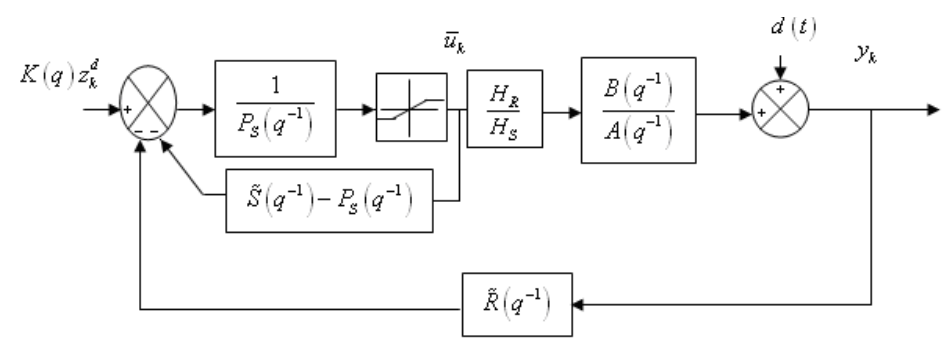

Figure 5: Diagram of flatness-based RST controller with taking in consideration of anti-saturation

Finally, the new control law of the flatness-based polynomial controller in the presence of saturation (47) is given by the expression:

$$
P_{S}\left(q^{-1}\right) u_{k}=K(q) z_{k}^{d}-\tilde{R}\left(q^{-1}\right) y_{k}-\left(\tilde{S}\left(q^{-1}\right)+\exp \left(\frac{T_{e}}{\tau_{s a t}}\right)\right) \bar{u}_{k-1}
$$

In the following part, a comparative study between the two laws of RST polynomial controllers, RST flatness-based control and conventional RST polynomial control, is carried out by digital simulation by considering the case of the thermal process control.

\section{Application to the control of a thermal system}

\subsection{Modelling of the thermal system}

The thermal process whose simplified diagram is given by figure 6 , is constituted of a tube of constant volume $V\left[\mathrm{~m}^{3}\right]$ and of a heating resistance $R_{c}$ connected to a direct current power supply $u(t)$ applied to resistance for heating the air entering at a desired temperature by Joule effect $6, C\left[\mathrm{~J} . \mathrm{m}^{-3}{ }^{\circ} \mathrm{K}^{-1}\right]$ is the specific heat constant of air, $T_{E}\left[{ }^{\circ} K\right]$ the ambient temperature, $f_{j}\left[m^{3} \cdot s^{-1}\right]$ the air rate flow entering according to the aperture of the valve $j$. The purpose of the control approach is to regulate the temperature $T_{S}\left[{ }^{\circ} \mathrm{K}\right]$ of the outgoing air at the constant temperature, given that the air flows into the tube with an initial temperature $T_{E}\left[{ }^{\circ} K\right]$ and at the flow rate $f_{j}\left[m^{3} \cdot s^{-1}\right]$.

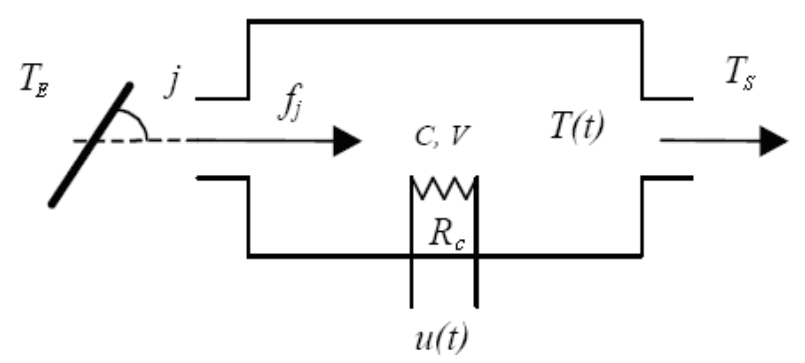

Figure 6: Simplified diagram of the thermal process

The flow rate signal is assumed piecewise constant and can be vary by changing the throttle position $j$.

The model of the thermal process, dependent, primarily of the valve position, if it is admitted that the temperature of the entering air remains constant, the transfer function of the model is given by $[10,16]$ : 


$$
G(p)=\frac{k e^{-\tau p}}{1+T_{c} p}
$$

where $k$ is the delay, $T$ is the time-constant of the process and $p$ is the Laplace operator. For an ambient temperature equal to $20 C$, the identified parameters of $G(p)$ are: $k=0.86, \tau=0.27 s$ and $T_{c}=$ o.49s $[10,11,16]$.

The corresponding discrete-time transfer function is then given by:

$$
G\left(q^{-1}\right)=\frac{0.0510 q^{-1}+0.3427 q^{-2}}{1-0.5421 q^{-1}}
$$

$T_{e}$ is the sampling period selected equal to $0.3 s$.

\subsection{Determination of the desired trajectory}

Let us consider that $B(1)=0.7875$, we choose to generate the trajectory expressed in continuous time $z^{d}(t)$ according to the following polynomial form:

$$
z^{d}(t)=\left\{\begin{array}{l}
\frac{y^{d}\left(t_{0}\right)}{B(1)}, \quad \text { if } 0 \leq t \leq t_{0} \\
\operatorname{Poly}(t), \quad \text { if } t_{0} \leq t \leq t_{f} \\
\frac{y^{d}\left(t_{f}\right)}{B(1)}, \quad \text { if } t \geq t_{f}
\end{array}\right.
$$

By taking the transition times $t_{0}=10 \mathrm{~s}$ and $t_{f}=20 \mathrm{~s}$, and choosing the polynomial Poly $(t)$, like reference trajectory between these two moments:

$$
\operatorname{Poly}(t)=\left(\begin{array}{ccc}
1 & 0 & 0
\end{array}\right)\left(M_{1}\left(t-t_{0}\right) c_{1}+M_{2}\left(t-t_{0}\right) c_{2}\right)
$$

The desired trajectory $z^{d}$ calculated is represented on figure 7 .

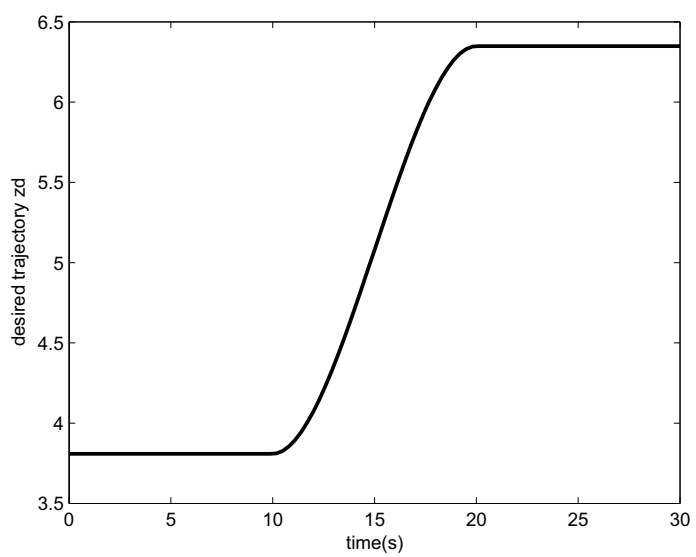

Figure 7: Desired trajectory $z^{d}(t)$

\subsection{Conventional RST controller by poles placement}

The tracking polynomial $K(q)$ is obtained by discretizing of the continuous model as third order system formed by the setting in cascade of two subsystems of which one is of second order, characterized 
by $\omega_{n}=2.5 \mathrm{rad} . \mathrm{s}^{-1}$ and $\xi=0.7$, and the other first order one having a time-constant $\tau=0.2 \mathrm{~s}$. It becomes then:

$$
K(q)=q^{3}-1.241 q^{2}+0.577 q-0.07808
$$

Simulations were carried out by considering the following conventional RST controller polynomials:

$$
\begin{gathered}
R\left(q^{-1}\right)=2.528-3.216 q^{-1}+1.015 q^{-2} \\
S\left(q^{-1}\right)=1+0.1724 q^{-1}-0.5306 q^{-2}-0.6419 q^{-3} \\
T\left(q^{-1}\right)=\frac{K\left(q^{-1}\right)}{B(1)}=-0.09915+0.7326 q^{-1}-1.575 q^{-2}+1.27 q^{-3}
\end{gathered}
$$

The simulation results show that the response of the thermal process presents an important tracking error. The thermal process presenting one zero unstable it is thus with no minimum of phase that explain the response of the system given in figure 8 .

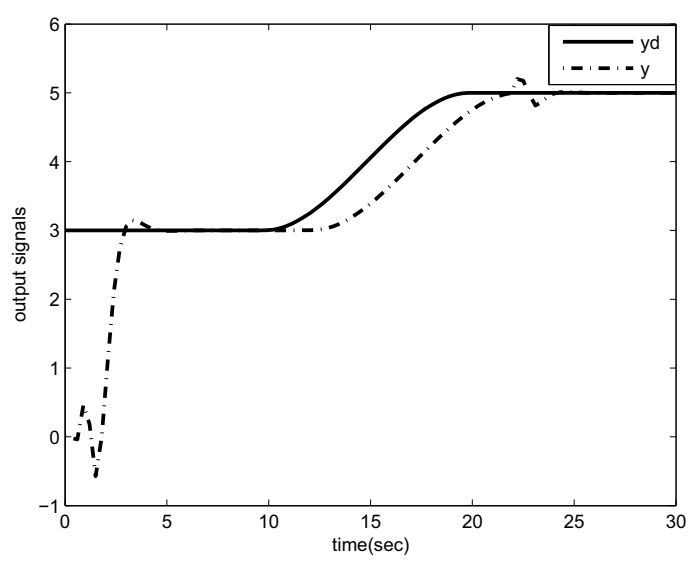

Figure 8: Conventional RST control: $y$ the real system output and $y^{d}$ the desired output

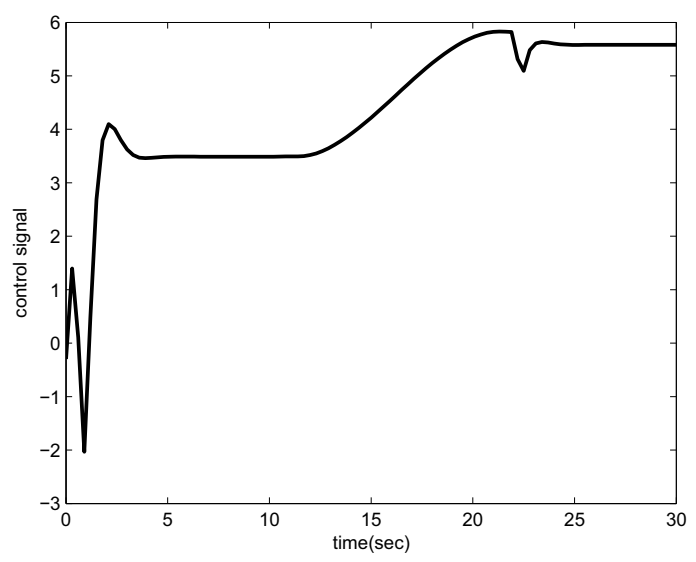

Figure 9: Conventional RST control: Control signal 


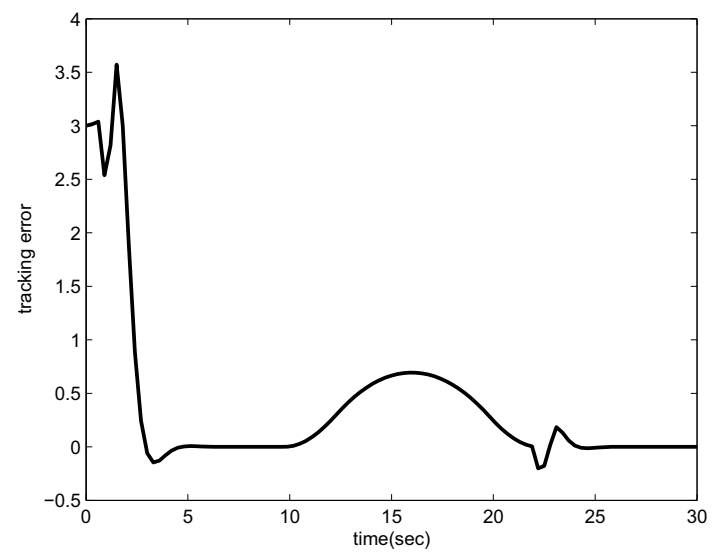

Figure 10: Conventional RST control: Tracking error

\subsection{Flatness-based RST controller}

The tracking polynomial $K(q)$ is selected starting from the discretization of the continuous tracking model considered as third order system. Consequently, we deduce the flatness-based RST regulator polynomials designed by flatness are bellow:

$$
\begin{gathered}
R\left(q^{-1}\right)=0.6554-0.3274 q^{-1} \\
S\left(q^{-1}\right)=1+0.2681 q^{-1}+0.2069 q^{-2}
\end{gathered}
$$

leading to the results of figures 11,12 and 13 .

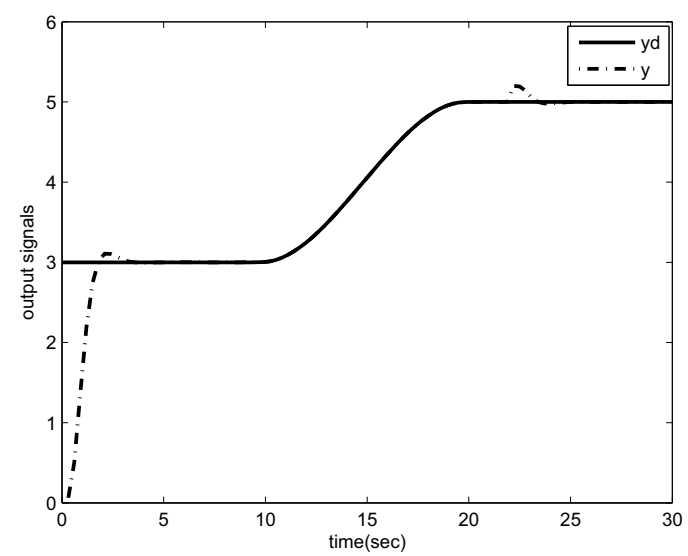

Figure 11: Flatness-based RST control: $y$ the real system output and $y^{d}$ the desired output

The results in figures 11, 12 and 13 underline the importance of the implementation of flatness-based controller in term of trajectory tracking. In fact, we notice in addition that the tracking error in the case of first approach RST is significant compared with that obtained by flatness-based controller. Thus, the flatness property is very interesting in terms of planning and tracking trajectory exploited for the development of a closed-loop system with high performances.

The results of figures 14 and 15 show that the controller obtained is robust considering the sensitivity functions which remain inside the specified templates of robustness [4]. In addition, we underline the 


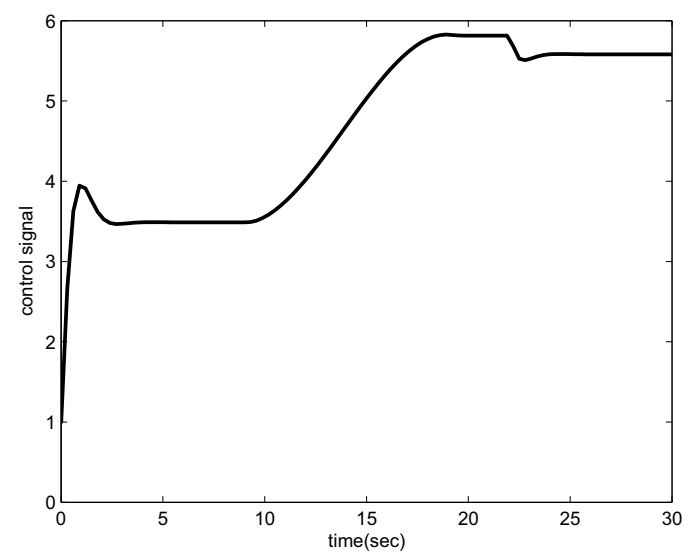

Figure 12: Flatness-based RST control: Control signal

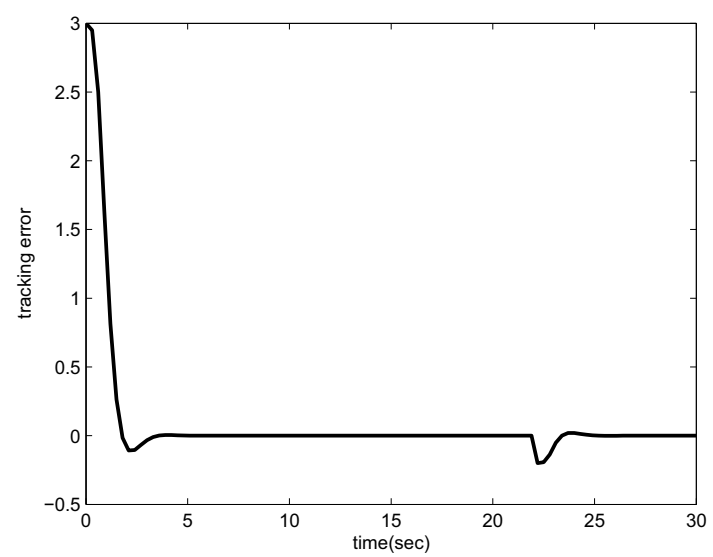

Figure 13: Flatness-based RST control: Tracking error

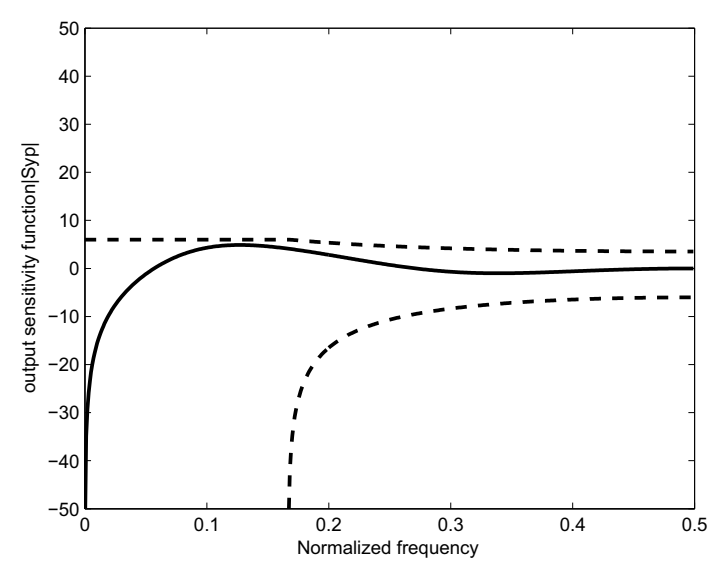

Figure 14: Output sensitivity function $|S y d|$ case of output disturbance 


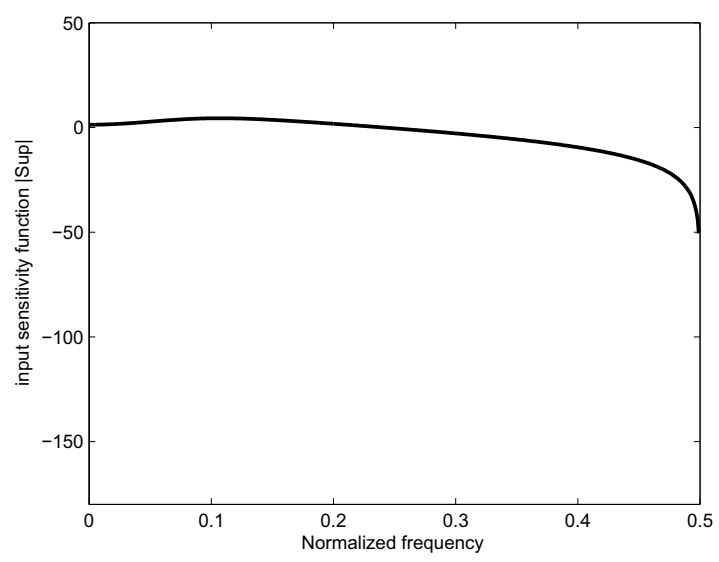

Figure 15: Input sensitivity function $|S u d|$ case of input disturbance

robustness of such controller related to the static disturbance and the high frequency noises rejection is clearly guaranteed. Moreover, the input sensitivity function presents attenuation in the high frequencies which define an elimination of noises in the input.

\section{Conclusion}

In conclusion, the study of the thermal process control shows that flatness-based RST regulator ended in a regulation of the robust and powerful type RST in term of tracking trajectories compared with such obtained by the use of the conventional polynomial RST controller. The design of flatness-based RST controller, depending on the choice of the poles of the tracking dynamic system and on the desired trajectory, allowed the satisfaction of the templates imposed by the typical values of the robustness margins.

\section{Bibliography}

[1] M. Fliess, J. Lévine, Ph. Martin and P. Rouchon, Sur les systčmes non linéaires différentiellement plats, Compte Rendu de l'Académie des Sciences de Paris, Série I, 315, pp. 619-624, 1992.

[2] M. Ayadi, Contribution ŕ la commande des systčmes linéaires plats de dimension finie, $\mathrm{PhD}$ Thesis, Institut National Polytechnique de Toulouse, 2002.

[3] T. Kailath, Linear systems, Prentice Hall, 1980.

[4] I. Landau, R. Lozano and M. M’Saad, Adaptative control, Springer-Verlag, London, 1998.

[5] F. Rotella, F J. Carrillo and M. Ayadi, Polynomial controller design based on flatness, Kybernetica Special Issue on System Structure and Control, Vol. 38, n5, pp. 571-584.

[6] A. Oustaloup, La robustesse. Analyse et synthčse de commandes robustes, Hermčs, 1994.

[7] M. Fliess and R. Marquez, Towards a module theoretic approach to discrete time linear predictive control, 14th International Symposium on Mathematical Theory of Networks and Systems, MTNS'2004, Perpignan, 2001. 
[8] M. M'Saad M. and J. Chebassier, Commande adaptative des systčmes, Techniques de l'Ingénieur, Vol. S2, nº 7426 , pp. 1-25, Paris, 1999.

[9] P. Borne, G. Dauphin-Tanguy, J. P. Richard, F. Rotella and I. Zambettakis, Commande et optimisation des processus, Edition Technip, Paris, 1990.

[10] S. Médar, Supervision et reconfiguration de la commande des systčmes dynamiques en présence de variations dans les conditions de fonctionnement, $\mathrm{PhD}$ Thesis, Institut National Polytechnique de Toulouse, 2002.

[11] F. Rotella, F. J. Carrilo and M. Ayadi, Digital flatness-based robust controller applied to a thermal process, IEEE International Conference on Control Application, pp. 936-941, Mexico, 2001.

[12] K. J. Aström and B. Wittenlmark, Adaptative Control, Addison Wesley, United States, 1989.

[13] M. Ayadi, N. Langlois, M. Benrejeb and H. Chafouk, Flatness-based robust adaptative polynomial controller for a diesel engine model, Transaction on Systems, Signals \& Devices, Vol. 2, n ${ }^{\circ}$, pp. 71-90, 2006.

[14] M. Fliess, J. Lévine, Ph. Martin and P. Rouchon, On Differentially Flat non Linear Systems, IFACSymposium NOLCOS'92, pp. 408-412, Bordeaux ,1992.

[15] R. Marquez, and M. Fliess, Linear Predictive Control Revisited. A Flatness Based Approach, European Control Conference, ECC'99, Karlshrule, 1999.

[16] $\mathrm{Ph}$. Charbonnaud, J. F. Carrilo and S. Médar, Robust control reconfiguration of a thermal process with multiple operating modes,IEEE transaction of control systems technology, Vol. 11, n $4,2003$.

[17] J.Y. Dieulot, I. Thimoumi, F. Colas and R. Béarée, Numerical Aspects and Performances of Trajectory Planning Methods of Flexible Axes, International Journal of Computers, Communications and Control, Vol.I, n4, pp. 35-44, 2006.

[18] J. Lévine, On the necessary and sufficent conditions for differential flatness, $6^{\text {th }}$ IFAC Symposium on Nonlinear Control Systems, NOLCOS'04, pp. 463-468, Stuttgart, 2004.

[19] H. Sira-Ramirez and S.K. Agrawal, Differentially flat systems, Marcel Dekker, 2004.

Hajer Gharsallaoui was born in 1979 and received the Engineer Diploma degree in 2004 and the Master Degree in Automatic Control in 2005 from the Ecole Nationale d'Ingénieurs de Tunis (ENIT). She is currently preparing the Ph.D. Degree in Automatic Control within the framework of LAGIS-EC-Lille and LARA-ENIT cooperation. Her research is related to Accommodation Control and FTC Control, diagnostic of flat system, and adaptive control.

Mounir Ayadi graduated from the Ecole Nationale d'Ingénieurs de Tunis in 1998 and received his $\mathrm{PhD}$ degree in Automatic Control from the Institut National Polytechnique de Toulouse in 2002. He was a post-doctoral fellow at the Ecole Supérieure d'Ingénieurs en Génie Electrique de Rouen in 2003. He is currently Maître-Assistant at the Ecole Nationale d'Ingénieurs de Tunis. His research interests are in the area of control system theory, predictive and adaptive control, and flat systems.

Mohamed Benrejeb was born in Tunisia in 1950. He obtained the Diploma of "Ingénieur IDN" (French "Grande Ecole") in 1973, the Master degree of Automatic Control in 1974, the PhD in Automatic Control of the University of Lille in 1976 and the DSc of the same University in 1980. He is currently a full Professor at the Ecole Nationale d'Ingénieurs de Tunis and an invited Professor at the Ecole Centrale de Lille. His research interests are in the area of analysis and synthesis of complex systems based on classical and non conventional approaches. 
Pierre Borne received the Master degree of Physics in 1967, the Masters of Electronics, of Mechanics and of Applied Mathematics in 1968. The same year he obtained the Diploma of "Ingénieur IDN" (French "Grande Ecole"). He obtained the PhD in Automatic Control of the University of Lille in 1970 and the DSc of the same University in 1976. He became Doctor Honoris Causa of the Moscow Institute of Electronics and Mathematics (Russia) in 1999, of the University of Waterloo (Canada) in 2006 and of the Polytechnic University of Bucarest (Romania). He is author or co-author of about 200 Journal articles and book chapters, and of 34 plenary lectures and of more than 250 communications in international conferences. He has been the supervisor of $71 \mathrm{PhD}$ thesis and is author of 20 books. He is Fellow of IEEE and has been President of the IEEE/SMC society in 2000 and 2001 .He is Vice-Chair of the IFAC TC on Large Scale Complex Systems. He is presently Professor "de classe exceptionnelle" at the Ecole Centrale de Lille and Director of the national french pluriformations group of research in Automatic Control. 